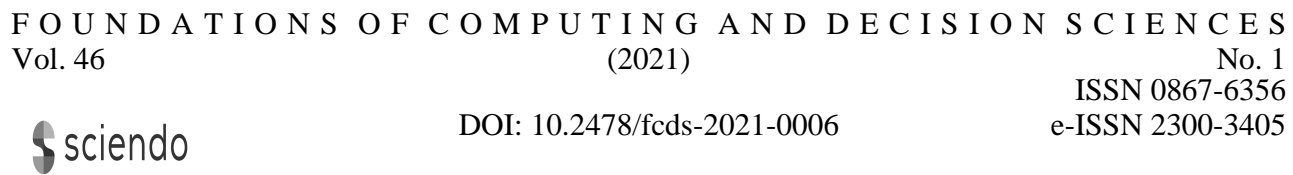

\title{
Revealed Comparative Advantage Method for Solving Multicriteria Decision-making Problems
}

\author{
Joseph Gogodze*
}

\begin{abstract}
This study proposes and analyzes a new method for the post-Pareto analysis of multicriteria decision-making (MCDM) problems: the revealed comparative advantage (RCA) assessment method. An interesting feature of the suggested method is that it uses the solution to a special eigenvalue problem and can be considered an analog/modification in the MCDM context of well-known ranking methods including the authority-hub method, PageRank method, and so on, which have been successfully applied to such fields as economics, bibliometrics, web search design, and so on. For illustrative purposes, this study discusses a particular MCDM problem to demonstrate the practicality of the method. The theoretical considerations and conducted calculations reveal that the RCA assessment method is self-consistent and easily implementable. Moreover, comparisons with wellknown tools of an MCDM analysis shows that the results obtained using this method are appropriate and competitive. An important particularity of the RCA assessment method is that it can be useful for decision-makers in the case in which no decision-making authority is available or when the relative importance of various criteria has not been preliminarily evaluated.
\end{abstract}

Keywords: Decision-making problem, revealed comparative advantage, multicriteria optimization

\section{Introduction}

The multicriteria formulation is a typical starting point for theoretical and practical analyses of decision-making problems. The concept of Pareto optimality and a vast arsenal

* Georgian Technical University, 77, Kostava Str., 0160, Tbilisi, Georgia; Email: sosogogodze@,gtu.ge 
of different Pareto-optimization methods are used for decision-making purposes. However, unlike single-objective optimizations, a characteristic feature of Pareto optimality is that the set of Pareto-optimal (efficient) alternatives is generally large. In addition, all these Paretooptimal alternatives must be considered mathematically equal. Correspondingly, the problem of choosing a specific Pareto-optimal alternative for implementation becomes relevant because the final decision must typically be unique, and additional factors must be considered to aid a decision-maker in the selection of specific or more favorable alternatives from the set of Pareto-optimal solutions. Thus, the solution to a multicriteria decision-making (MCDM) problem generally involves two stages: the Pareto-optimization stage and postPareto analysis stage (i.e., choosing one appropriate Pareto-solution from a set of efficient alternatives, which is also called post-Pareto optimality analysis).

Many Pareto-optimization methods have been proposed, and a vast amount of literature exists on this topic. However, a limited amount of literature has been devoted to the postPareto analysis stage. Usually, this literature is presented in the context of multi-objective decision-making (MODM) problems, and we point out below (without any details) only a few of them: the compromise programming, goal programming, utility function approaches $[21,35,40,42]$, marginal rate of substitution approach [29], nonnumerical ranking preference method [12], Pareto set clustering method [1, 36, 37], greedy reduction algorithm [39], restricting weight method [26], local search with achievement scalarizing function [31], hybrid method based on fuzzy logic and evolutionary algorithms [22], nonuniform weight generator method [8], sweeping cones method [9], and so on. For an overview of some postPareto analysis methods (in the MODM context), see [11]. Literature regarding the postPareto analysis directly in the MCDM context is missing to the best of our knowledge, but most of the mentioned methods can be adapted accordingly. Moreover, the well-known methods for the solution to an MCDM problem (e.g., AHP [33], ANP [34], ELECTRE [32], PROMETHEE [6], TOPSIS [18], VIKOR [30], SAW [23], WPM [38], MACBETH [4], MAUT [14], MOORA [7] RPA [3], etc.) usually provide the corresponding rankings of the alternatives and, hence, allow the post-Pareto stage of analysis. It is clear from this short overview of the literature that the process of post-Pareto analysis method development is far from complete and that its further development will be useful for applications.

The purpose of this study is to develop a new post-Pareto analysis method for the MCDM problem solution and illustrate its ability using the solution of an engineering test problem. The suggested method can be briefly described as follows: for any MCDM problem $\langle A, C\rangle$, where $A$ is the set of alternatives and $C$ is the set of criteria, the assessment function $J: A \times C \rightarrow \mathbb{R}$ can naturally be defined (it evaluates each criterion on each alternative). In this study, based on the assessment function $J$, a special procedure was defined, allowing alternatives to be ranked. The best alternative found using this ranking procedure can be declared a 'solution' of the MCDM problem. Because the procedure directly considers (through the assessment function $J$ ) the natural relationship between the alternatives and criteria for the analyzed MCDM problem, the alternative declared above as a 'solution' can be treated (only subjectively) as a "true solution" obtained in the post-Pareto analysis stage for the MCDM problem. The method is called the revealed comparative 
advantage (RCA) assessment method ${ }^{1}$ because a preliminary assessment of the comparative advantages of alternatives is conducted during its implementation. Moreover, the method defines the ranking of alternatives based on the solution to a special eigenvalue problem and can be considered an analog/modification in the MCDM context of well-known ranking methods (e.g., the authority-hub method, PageRank method, and others), which have been successfully applied to numerous fields, such as economics, bibliometrics, web search design, and so on, for solutions to the "essentially big size" problems (see e.g., [5, 16, 17, 25, 27]). Thus, we expect that the suggested method can be an effective tool for solving MCDM problems (especially with large sets of alternatives or criteria).

We illustrate the RCA assessment method using a special example known in the scientific literature: the material-selection problem (see [15]). Material-selection problems are a very important area of MCDM engineering applications and attract the attention of many researchers. In particular, the considered example was investigated previously by several researchers using various methods and can be considered a very convenient benchmarking problem for conducting comparative analyses of different methods (see [13, $15,20,23,24])$. For the example under consideration, we can compare the solution obtained using the RCA assessment method with solutions obtained using the following methods: MOORA, MMOORA, RPA, FLA (see [23]), Wpm [15], VIKOR, and CVIKOR, [20]. For the comparison of the results obtained using different methods, we used Spearman's rank correlation method and showed that the solution obtained using the offered method is acceptable and yields a competitive ranking of alternatives for the considered MCDM. This illustration reveals the viability and applicability of the RCA assessment method for MCDM problems.

The rest of this paper is structured as follows. In Section 2, the RCA assessment method is described. Section 3 considers an illustrative example. Finally, Section 4 presents the discussion and conclusion of the study.

\section{Method}

Alternatives are denoted by $A=\left\{a_{1}, \ldots, a_{m}\right\}$, and the set of criteria is denoted by $C=\left\{c_{1}, \ldots, c_{n}\right\} ; c_{i}: A \rightarrow \mathbb{R}$, where $i \in \mathbb{N}(n)=\{1, \ldots, n\}$. The MCDM problem considers the ordered pair $\langle A, C\rangle$. The criteria can be assumed to be normalized, such that a lower value is preferable for each criterion. The goal of the decision-making procedure is to minimize all criteria simultaneously [28]. Further, we adhere to this assumption.

Additionally, $A$ is the set of admissible alternatives, and map $\vec{c}=\left(c_{1}, \ldots, c_{n}\right): A \rightarrow \mathbb{R}^{n}$ is the criterion map (correspondingly, $\vec{c}(A) \subset \mathbb{R}^{n}$ is the set of admissible values of the criteria). An alternative, $a_{*} \in A$, is Pareto optimal (i.e., efficient) if no $a \in A$ exists, such that $c_{j}(a) \leq c_{j}\left(a_{*}\right)$ for all $j \in \mathbb{N}(n)$ and $c_{k}(a)<c_{k}\left(a_{*}\right)$ for some $k \in \mathbb{N}(n)$. The set of all efficient alternatives is denoted as $A_{e}$ and is called the Pareto set. Correspondingly, $f\left(A_{e}\right)$ is called the efficient frontier. Without loss of generality, we assume further that an MCDM

${ }^{1}$ The term "revealed comparative advantage" was introduced in [2] and has been widely used in economics for comparative analyses of countries from the international trade perspective. 
problem $\left\langle A, C>\right.$ is given, where $A=\left\{a_{1}, \ldots, a_{m}\right\}$ and $C=\left\{c_{1}, \ldots, c_{n}\right\}$, and that the goal of the decision-making procedure is to minimize all criteria simultaneously.

The MCDM problem $\langle A, C\rangle$ is associated with a special assessment function and a special matrix, which can be used successfully to determine the final solution. We introduce the following notation. First, we define the function $J: A \times C \rightarrow \mathbb{R}$ by the equality $J(a, c)=c(a) \forall a \in A, c \in C$. Then, we call $J: A \times C \rightarrow \mathbb{R}$ a general assessment function for the MCDM problem $\langle A, C\rangle$. Further, we assume the following:

$$
\left\{\begin{array}{c}
\mathrm{J}(\mathrm{a}, \mathrm{c}) \geq 0, \forall \mathrm{a} \in \mathrm{A}, \forall \mathrm{c} \in \mathrm{C}, \\
I_{C}(a)=\sum_{c \in C} J(a, c)>0 \forall a \in A, \\
I_{A}(c)=\sum_{a \in A} J(a, c)>0 \forall c \in C,
\end{array}\right.
$$

which does not significantly reduce the generality of our considerations in the sense of applicability to practical MCDM problems. We define the function $J_{R C A}: A \times C \rightarrow \mathbb{R}$ as follows:

$$
J_{R C A}(a, c)=\iota(a, c) / \iota_{C}(a) \iota_{A}(c) \forall a \in A, c \in C,
$$

where $\iota_{C}(a)=I_{C}(a) / I_{A C}, \iota_{A}(c)=I_{A}(c) / I_{A C}, I_{A C}=\sum_{a \in A, c \in C} J(a, c), \iota(a, c)=J(a, c) / I_{A C}$, for any $a \in A, c \in C$. We call $J_{R C A}$ the RCA assessment function for the MCDM problem $<A, C>$.

Next, we introduce the following quantities:

$$
u_{J}(a)=\sum_{c \in C} M_{J}(a, c), d_{J}(c)=\sum_{a \in A} M_{J}(a, c), M_{J}(a, c)= \begin{cases}1, & J_{R C A}(a, c)<1, \\ 0, & J_{R C A}(a, c) \geq 1,\end{cases}
$$

and define the following matrices:

$$
\begin{gathered}
W_{C}=\left[W_{C}(a, c)\right]_{(a, c) \in A \times c} ; W_{C}(a, c)=M_{J}(a, c) / u_{J}(a) \quad \forall(a, c) \in A \times C ; \\
W_{A}=\left[W_{A}(a, c)\right]_{(a, c) \in A \times C} ; W_{A}(a, c)=M_{J}(a, c) / d_{J}(c) \quad \forall(a, c) \in A \times C .
\end{gathered}
$$

Next, we introduce the vectors $e \in \mathbb{R}^{m}$ and $s \in \mathbb{R}^{n}$, which are defined by the following equalities: $e=\kappa_{e} W_{C} s$ and $s=\kappa_{s} W_{A} e$, where $\kappa_{e}, \kappa_{s}>0$ are constants. The vectors $e \in \mathbb{R}^{m}$ and $s \in \mathbb{R}^{n}$ can be called the effectiveness vector for alternatives and the significance vector for criteria, respectively. These equations reflect a simple idea: the effectiveness of alternatives is directly proportional to the weighted sum of the criteria significance, and the significance of the criteria is directly proportional to the weighted sum of the alternative effectiveness. Consequently, $W_{C A} e=\lambda e, W_{A C} s=\lambda s$, where $\lambda=\left(\kappa_{e} \kappa_{s}\right)^{-1}, W_{C A}=W_{C} W_{A}$, and $W_{A C}=W_{A} W_{C}$. This indicates that $\lambda$ is an eigenvalue and $e \in \mathbb{R}^{m}$ and $s \in \mathbb{R}^{n}$ are the corresponding eigenvectors. Following [19], we select eigenvectors corresponding to the second-largest eigenvalue. Thus, we obtain the vectors $e \in \mathbb{R}^{m}$ and $s \in \mathbb{R}^{n}$, which are rating vectors for alternatives and criteria, respectively. 
Further, the matrix $W_{C A}\left(W_{A C}\right)$ is called an RCA matrix for alternatives (for criteria), and its eigenvector corresponding to the second-largest eigenvalue is called an RCA-rating vector for alternatives (criteria) (see also uses of eigenvectors in ranking procedures). Hence, we can establish the following procedure for solving MCDM problems, which is the RCA assessment method:

(i) The RCA matrix for alternatives is calculated for the MCDM problem $\langle A, C\rangle$;

(ii) The RCA-rating vector $r^{r c a}$ for the alternatives is determined, and the alternative from the Pareto set, $A_{e}$, that is best according to the $r^{r c a}$ ranking is declared the solution of the considered MCDM problem $\langle A, C\rangle$.

Various modifications of the described construction are possible. Moreover, previous experience (see e.g., $[5,16,17,19,25,27])$ indicates that the corresponding analogs of the described method may be useful for solving large-scale MCDM problems (i.e., with large sets of alternatives and/or criteria).

\section{Numerical Example}

This section discusses the selected numerical example to demonstrate the practicality of the method. The considered example is the problem of selecting material for the mast of a sail-

Table 1. Decision matrix for selecting material for a sailing boat mast

\begin{tabular}{|c|c|c|c|c|c|}
\hline \multirow{3}{*}{ \# } & \multirow{3}{*}{ Material } & \multicolumn{4}{|c|}{ Criteria (beneficial) } \\
\hline & & SS & SM & CR & $\mathrm{CC}$ \\
\hline & & 1 & 2 & 3 & 4 \\
\hline 1 & AISI 1020 & 35.9 & 26.9 & 1 & 5 \\
\hline 2 & AISI 1040 & 51.3 & 26.9 & 1 & 5 \\
\hline 3 & ASTM A242 type 1 & 42.3 & 27.2 & 1 & 5 \\
\hline 4 & AISI 4130 & 194.9 & 27.2 & 4 & 3 \\
\hline 5 & AISI 316 & 25.6 & 25.1 & 4 & 3 \\
\hline 6 & AISI 416 heat treated & 57.1 & 28.1 & 4 & 3 \\
\hline 7 & AISI 431 heat treated & 71.4 & 28.1 & 4 & 3 \\
\hline 8 & AA 6061 T6 & 101.9 & 25.8 & 3 & 4 \\
\hline 9 & AA 2024 T6 & 141.9 & 26.1 & 3 & 4 \\
\hline 10 & AA 2014 T6 & 148.2 & 25.8 & 3 & 4 \\
\hline 11 & AA 7075 T6 & 180.4 & 25.9 & 3 & 4 \\
\hline 12 & $\mathrm{Ti}-6 \mathrm{Al}-4 \mathrm{~V}$ & 208.7 & 27.6 & 5 & 1 \\
\hline 13 & Epoxy-70\% glass fabric & 604.8 & 28.0 & 4 & 2 \\
\hline 14 & Epoxy $-63 \%$ carbon fabric & 416.2 & 66.5 & 4 & 1 \\
\hline 15 & Epoxy $-62 \%$ aramid fabric & 637.7 & 27.5 & 4 & 1 \\
\hline
\end{tabular}

Notes: Source: [6]; Strength (SS); Specific modulus (SM); Corrosion resistance (CR), scale: $1=$ poor, $2=$ fair, $3=$ good, $4=$ very good, and $5=$ excellent; Cost category $(\mathrm{CC})$, scale: $1=$ very high, $2=$ high, $3=$ moderate, $4=$ low, and $5=$ very low.

boat (see [13, 15, 20, 23, 24]). The choice must be made from 15 alternative materials, which were evaluated using the following criteria: strength (SS), specific modulus (SM), corrosion resistance (CR), and cost category (CC) (see [15] for details). The corresponding decision- 
making matrix is given in Table 1, where all criteria are beneficial. After transformation (the upper-lower-bound approach was used [28]), we obtained a decision matrix with nonbeneficial criteria (Table 2), which yields the Pareto set $A_{e}=\{2,3,4,7,9,11,12,13,14,15\}$. Table 3 presents the RCA matrix $W_{C A}$ and the corresponding RCA-rating vector $r^{r c a}$ (second eigenvalue, $\lambda=0.8578$ ).

Table 2. Normalized decision matrix for the material-selection problem

\begin{tabular}{|c|c|c|c|c|c|}
\hline & \multicolumn{4}{|c|}{ Normalized Criteria (nonbeneficial) } \\
\hline & & 1 & 2 & 3 & 4 \\
\hline \multirow{15}{*}{$\begin{array}{l}\frac{n}{\pi} \\
\frac{\pi}{\bar{d}} \\
\frac{\pi}{\pi} \\
\sum\end{array}$} & 1 & 0.9832 & 0.9565 & 1.0000 & 0.0000 \\
\hline & 2 & 0.9580 & 0.9565 & 1.0000 & 0.0000 \\
\hline & 3 & 0.9727 & 0.9493 & 1.0000 & 0.0000 \\
\hline & 4 & 0.7234 & 0.9493 & 0.2500 & 0.5000 \\
\hline & 5 & 1.0000 & 1.0000 & 0.2500 & 0.5000 \\
\hline & 6 & 0.9485 & 0.9275 & 0.2500 & 0.5000 \\
\hline & 7 & 0.9252 & 0.9275 & 0.2500 & 0.5000 \\
\hline & 8 & 0.8753 & 0.9831 & 0.5000 & 0.2500 \\
\hline & 9 & 0.8100 & 0.9758 & 0.5000 & 0.2500 \\
\hline & 10 & 0.7997 & 0.9831 & 0.5000 & 0.2500 \\
\hline & 11 & 0.7471 & 0.9807 & 0.5000 & 0.2500 \\
\hline & 12 & 0.7009 & 0.9396 & 0.0000 & 1.0000 \\
\hline & 13 & 0.0537 & 0.9300 & 0.2500 & 0.7500 \\
\hline & 14 & 0.3619 & 0.0000 & 0.2500 & 1.0000 \\
\hline & 15 & 0.0000 & 0.9420 & 0.2500 & 1.0000 \\
\hline
\end{tabular}

Note: Pareto-optimal (efficient) alternatives are marked in gray. 
Table 3. RCA-matrix and the RCA-rating vector for the material-selection problem

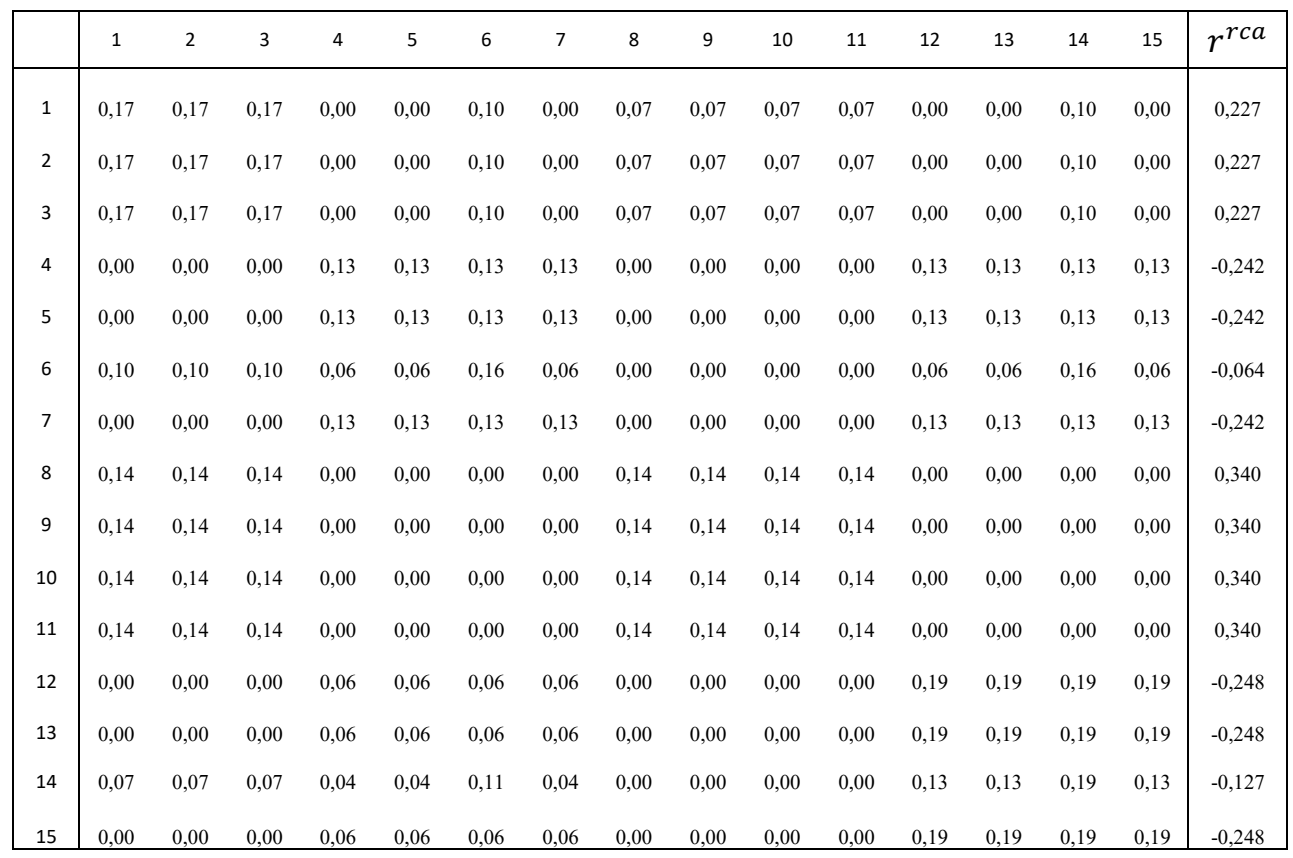

For the sake of comparison, Table 4 (see also its visualization in Figure 1) presents the ranks of the materials obtained using the RCA assessment method, $r^{r c a}$, and the previously used methods. The obtained results are also summarized based on Spearman's rank correlation analysis (see Table 5). All our numerical exercises were conducted on standard equipment (laptop with $2.59 \mathrm{GHz}, 8 \mathrm{~GB}$ RAM, and 64-bit operation system) in the MATLAB environment, without optimizing the code.

Table 4. Materials ranked by comparable methods

\begin{tabular}{l|ccccccccccccccc}
\hline Method $\backslash$ Material & 1 & 2 & 3 & 4 & 5 & 6 & 7 & 8 & 9 & 10 & 11 & 12 & 13 & 14 & 15 \\
\hline MOORA, [23] & 14 & 15 & 13 & 12 & 4 & 7 & 6 & 11 & 10 & 9 & 5 & 8 & 2 & 3 & 1 \\
MMOORA, [23] & 14 & 15 & 13 & 12 & 4 & 11 & 10 & 9 & 7 & 6 & 8 & 5 & 2 & 3 & 1 \\
RPA, [23] & 14 & 13 & 12 & 15 & 4 & 11 & 10 & 9 & 8 & 7 & 6 & 2 & 3 & 1 & 5 \\
FLA, [23] & 14 & 13 & 15 & 4 & 11 & 9 & 10 & 8 & 12 & 7 & 6 & 5 & 3 & 2 & 1 \\
Wpm, [15] & 14 & 13 & 15 & 11 & 10 & 9 & 8 & 7 & 2 & 4 & 6 & 3 & 12 & 1 & 5 \\
CVIKOR, [20] & 12 & 6 & 9 & 4 & 15 & 14 & 11 & 13 & 8 & 10 & 5 & 7 & 2 & 1 & 3 \\
VIKOR, [20] & 14 & 11 & 13 & 4 & 15 & 10 & 5 & 12 & 7 & 9 & 6 & 8 & 2 & 1 & 3 \\
$r^{r c a}$ & 9 & 10 & 11 & 4 & 5 & 8 & 6 & 12 & 13 & 14 & 15 & 1 & 2 & 7 & 3 \\
\hline
\end{tabular}

Note: Pareto-optimal (efficient) alternatives are marked in gray. 


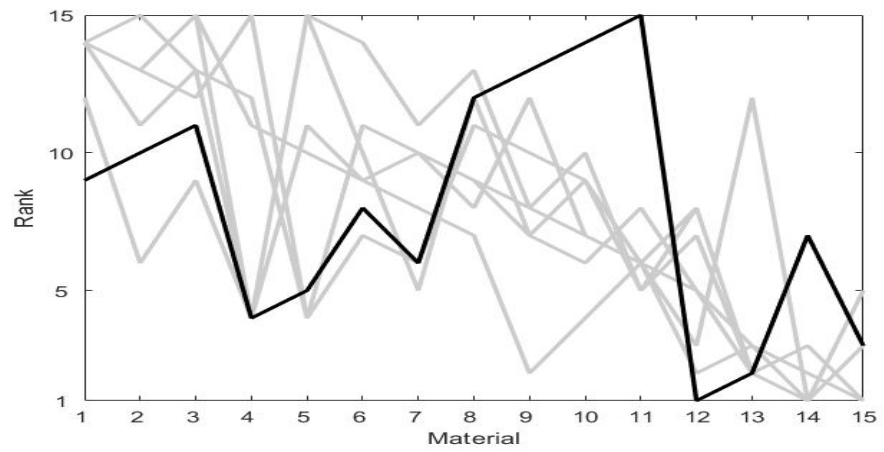

Figure 1. Rank comparison

Note: See material names in Table $1, r^{r c a}$ - ranking marked in dark gray

Table 5. Spearman's rank correlations

\begin{tabular}{|c|c|c|c|c|c|c|c|c|}
\hline & MOORA & MMOORA & RPA & FLA & Wpm & CVIKOR & VIKOR & $r^{r c a}$ \\
\hline MOORA & 1,000 & & & & & & & \\
\hline MMOORA & 0,871 & 1,000 & & & & & & \\
\hline RPA & 0,793 & 0,911 & 1,000 & & & & & \\
\hline FLA & 0,696 & 0,718 & 0,596 & 1,000 & & & & \\
\hline Wpm & 0,457 & 0,618 & 0,643 & 0,525 & 1,000 & & & \\
\hline CVIKOR & 0,325 & 0,375 & 0,339 & 0,657 & 0,239 & 1,000 & & \\
\hline VIKOR & 0,589 & 0,525 & 0,414 & 0,804 & 0,471 & 0,818 & 1,000 & \\
\hline$r^{r c a}$ & 0,421 & 0,396 & 0,307 & 0,471 & $-0,071$ & 0,279 & 0,354 & 1,000 \\
\hline
\end{tabular}

\section{Discussion and Conclusion}

The solution for MCDM problems has received considerable attention from researchers for quite a long time, and the main tool for finding a solution is Pareto optimization. However, the attention of researchers has recently been increasingly focused on post-Pareto analysis issues. This circumstance is connected to the fact that the set of Pareto solutions is usually large in the sense of the element number, but for implementation, only one solution must be allocated. In this brief note, a novel method for solving MCDM problems, the RCA assessment method, was proposed. In the framework of the method, we built an appropriate ranking method that conducts a post-Pareto analysis, choosing the best-ranked alternative from the Pareto set as a "true solution."

To demonstrate the viability and suitability of this method for application, the RCA assessment method was illustrated using a numerical example. On the example under consideration, the method has been compared with seven well-known methods: MOORA, MMOORA, RPA, FLA, Wpm, VIKOR, and CVIKOR. The ranking of alternatives obtained using the RCA assessment method does not generally correlate significantly with the rankings obtained using other methods (see the numerical example in Section 3, Table 5). 
However, the final solution of the MCDM problem determined using the method seems quite adequate and competitive. For example, in the considered numerical example, the suggested method chose material from the cheapest cost category with a lower strength and specific modulus but a higher corrosion resistance index. Such a choice can be considered as an acceptable engineering solution. Hence, the RCA assessment method, as a post-Pareto analysis tool, can be expected to generate "more original/novel" Pareto solutions than wellknown traditional methods. Of course, this assumption requires a deeper empirical confirmation in future research.

Some criticism may exist regarding the offered method because its implementation must solve the eigenvalue problem, which is a complex mathematical problem, especially for large-scale problems. However, well-known methods exist for overcoming this complication, such as iterative procedures and/or methods of matrix perturbation, that have worked well in a variety of applications (see, e.g., [5, 16, 17, 25, 27]). Using these methods effectively overcomes the difficulty of finding a solution for large-scale eigenvalue problems and makes the RCA assessment method acceptable for "essence big-scale" problems.

The RCA assessment method is easy and flexible for implementation and, as mentioned, it can be modified to acquire the necessary properties for solving MCDM problems with large sets of alternatives and/or criteria. The method is useful when no decision-making authority is available or when the relative importance of various criteria has not been previously evaluated (i.e., criteria were not previously weighted according to importance). However, the suggested method has another not-yet-mentioned opportunity that should be addressed in future research. Namely, because the method also allows ranking the criteria, it can also identify a "leading criterion." Obtained this way, the "leading criterion" can be used to develop a two-level Pareto-optimization procedure that can be useful in various applications.

\section{References}

[1] Aguirre, O. and Taboada, H. A clustering method based on dynamic self-organizing trees for post-pareto optimality analysis. Procedia Computer Science, 6, 2011,195-200.

[2] Balassa, B. Trade liberalisation and "revealed" comparative advantage, The Manchester school, 33, 1965, 99-123.

[3] Balf, F. R. Ranking Efficient Units by Regular Polygon Area (RPA) in DEA, International Journal of Industrial Mathematics, 3(1), 2011, 41-53.

[4] Bana E Costa C.A., and Vansnick J.C. The MACBETH Approach: Basic Ideas, Software, and an Application. In: Meskens N., Roubens M. (eds) Advances in Decision Analysis. Mathematical Modelling: Theory and Applications, vol 4, 1999, Springer, Dordrecht.

[5] Bollen, J., Rodriquez, M.A., and Van de Sompel, H. Journal status. Scientometrics, 69, 2006, 669-687.

[6] Brans, J. L'ingenierie de la decision; Elaboration d'instruments d'aide a la decision. La methode PROMETHEE, in L'aide a la decision: Nature, Instruments et Perspectives d'Avenir, R. Nadeau and M. Landry, Eds. Quebec, Canada: Presses de l'Universite Laval, 1982, pp. 183-213. 
[7] Brauers, W. K. M., Zavadskas, E. K. The MOORA method and its application to privatization in a transition economy. Control and Cybernetics, 35(2), 2006, 445.

[8] Carrillo VM, Taboada H.. A post-Pareto approach for multi-objective decision making using a non-uniform weight generator method. Procedia Computer Science. 12:1, 2012,16-121.

[9] Carrillo VM, Taboada H. A sweeping cones technique for post pareto analysis. In Industrial and Systems Engineering Research Conference, 2013.

[10] Carrillo VM, Taboada H., A General Iterative Procedure of the Non-Numerical Ranking Preferences Method for Multiple Objective Decision Making. Procedia Computer Science. Procedia Computer Science. 12, 2012,135-139.

[11] Carrillo, V.M. Post-Pareto optimality methods for the analysis of large Pareto sets in multi-objective optimization, 2013.

[12] Carrillo, V.M., Aguirre, O. and Taboada, H. Applications and performance of the nonnumerical ranking preferences method for post-Pareto optimality. Procedia Computer Science, 6, ., 2011, 243-248.

[13]Chatterjee, P., Athawale, V.M., and Chakraborty, S. Selection of materials using compromise ranking and outranking methods. Materials \& Design, 30, (2009), 40434053.

[14]Churchman, C.W., Ackoff, R.L., Arnoff, E.L. Introduction to Operations Research, A John Wiley and Sons, Ltd., Publication., New York 1957

[15]Farag, M.M. Quantitative methods of materials selection, In: Kutz M. (Eds) Handbook of Materials Selection, John Wiley \& Sons. 2002.

[16] Gleich, D.F. PageRank beyond the Web. SIAM Review, 57, 2015, 321-363.

[17] Hidalgo, C.A. and Hausmann, R. The building blocks of economic complexity. Proceedings of the National Academy of Sciences, 106, 2009, 10570-10575.

[18]Hwang, C.-L., Lai Y.-J., and Liu, T.-Y.. A new approach for multiple objective decision making, Comput. Oper. Res., vol. 20, no. 9, , 1993, 889-899.

[19]Inoua, S. A simple measure of economic complexity. arXiv preprint,2016, arXiv:1601.05012.

[20]Jahan, A., Mustapha, F., Ismail, M.Y., Sapuan, S.M., and Bahraminasab, M. A comprehensive VIKOR method for material selection. Materials \& Design, 32, (2011), 1215-1221.

[21] Jones, D., Tamiz, M. A review of goal programming. In Multiple criteria decision analysis, 2016, 903-926.

[22] Kacen, I., Hammadi, S., Borne, P. Pareto- optimality Approach on Uniform Design and Fuzzy Evolutionary algorithms for Flexible Job-shop Scheduling. Problems. Systems, Man and Cybernetics, 2002 IEEE International Conference, 2003. 
[23] Karande, P. and Chakraborty, S. Application of multi-objective optimization on the basis of ratio analysis (MOORA) method for materials selection. Materials \& Design, 37, (2012), 317-324.

[24]Khabbaz, R., Sarfaraz, B., Dehghan Manshadi, A., Abedian, R. Mahmudi. A simplified fuzzy logic approach for materials selection in mechanical engineering design. Materials \& Design, 30, (2009), 687-697.

[25] Kleinberg, J.M. Authoritative sources in a hyperlinked environment. Journal of the ACM (JACM), 46, (1999), 604-632.

[26] Korhonen, H. Restricting weights in value efficiency Analysis, European Journal of Operation Research,126, 2000, 175-188.

[27] Langville, A.N., Meyer, C.D. Deeper inside page rank. Internet Mathematics, 1, 2004, 335-380.

[28] Marler, R.T. and Arora, J.S. Function-transformation methods for multi-objective optimization. Engineering Optimization, 37, 2005, 551-570.

[29] Mietinen,K. Nonlinear Multi-objective Optimization. Boston: Kluer Academic Publishers, 1999.

[30] Opricovic S., Tzeng, G., Extended VIKOR method in comparison with outranking methods, European Journal of Operational Research, vol. 178, no. 2, , 2007, 514-529.

[31] Padhye, N., Kalia, S. and Deb, K. Multi-Objective Optimization and Multi-criteria Decision Making For FDM Using Evolutionary Approaches, 2009.

[32]Roy, B., Classement et choix en pr'esence de points de vue multiples (la m'ethode ELECTRE). Revue Francaise d'Informatique et de Recherche Op'erationnelle 8, 1968, $57-75$.

[33] Saaty, T. L. How to make a decision : the analytic hierarchy process, European journal of operational research, vol. 48, 1, , 1990, 9-26.

[34] Saaty, T. L., Decision making with dependence and feedback : The analytic network process, RWS publications Pittsburgh. 1996.

[35] Salukvadze, M. E.: Optimization of vector functionals. Part I: Programming of optimal trajectories (in Russian). Avtomatika i Telemekhanika, 8, 1971, 5-15.

[36] Taboada, H. \& Coit, D., Data Mining Techniques to Facilitate the Analysis of the ParetoOptimal Set for Multiple Objective Problems. In Proceedings of the Industrial Engineering Research Conference (IERC), Orlando, Florida, 2006.

[37] Taboada, H. and Coit, D. Post-Pareto Optimality Analysis to Efficiently Identify Promising Solutions for Multi-Objective Problems, Rutgers University ISE Working Paper 05-15, 2005.

[38] Triantaphyllou, E., Multi-criteria decision-making methods, in Multicriteria decision making methods: A comparative study. Dordrecht, The Netherlands: Kluwer Academic Publishers (now Springer)., 2000, pp. 5-21. 
[39] Venkat, V., Jacobson, S., Stori, J. 2004. A Post-Optimality Analysis Algorithm for Multi-Objective Optimization. Computational Optimization and Applications. 28, 357372.

[40] Yu P.L., Leitmann G. Compromise solutions, domination structures, and Salukvadze's solution. Journal of Optimization Theory and Applications, 13(3), 1974,362-378.

[41]Zadeh, L., Optimality and non-scalar-valued performance criteria, IEEE Transactions on Automatic Control, vol. 8, no. 1, pp. 59-60, 1963.

[42]Zeleny, M., Multiple criteria decision making. New York: McGraw-Hill, 1982.

Received 5.06.2020, Accepted 24.11.2020 